Article

\title{
Analysis of Flavonoids in Dalbergia odorifera by Ultra-Performance Liquid Chromatography with Tandem Mass Spectrometry
}

\author{
Xiangsheng Zhao ${ }^{1}$, Shihui Zhang ${ }^{1}$, Dan Liu ${ }^{2}$, Meihua Yang ${ }^{1,3}$ and Jianhe Wei ${ }^{1,3, *}$ \\ 1 Hainan Provincial Key Laboratory of Resources Conservation and Development of Southern Medicine, \\ Hainan Branch of the Institute of Medicinal Plant Development, Chinese Academy of Medical Sciences \& \\ Peking Union Medical College, Haikou 570311, China; xiangshengzhao@hotmail.com (X.Z.); \\ 13006070475@163.com (S.Z.); yangmeihua15@hotmail.com (M.Y.) \\ 2 School of Life Science and Engineering, Southwest University of Science and Technology, \\ Mianyang 621010, China; 15280986611@163.com \\ 3 Institute of Medicinal Plant Development, Chinese Academy of Medical Sciences \& Peking Union Medical \\ College, Beijing 100193, China \\ * Correspondence: wjianh@263.net; Tel.: +86-10-57833358
}

Received: 14 December 2019; Accepted: 15 January 2020; Published: 17 January 2020

check for updates

\begin{abstract}
Dalbergia odorifera, a traditional Chinese medicine, has been used to treat cardio- and cerebrovascular diseases in China for thousands of years. Flavonoids are major active compounds in D. odorifera. In this paper, a rapid and sensitive ultra-high performance liquid chromatography-triple quadrupole mass spectrometry method was developed and validated for simultaneous determination of 17 flavonoids in D. odorifera. Quantification was performed by multiple reaction monitoring using electrospray ionization in negative ion mode. Under the optimum conditions, calibration curves for the 17 analytes displayed good linearity $\left(r^{2}>0.9980\right)$. The intra- and inter-day precisions (relative standard deviations) were lower than 5.0\%. The limit of quantitation ranged from 0.256 to 18.840 $\mathrm{ng} / \mathrm{mL}$. The mean recovery range at three spiked concentrations was $94.18-101.97 \%$. The validated approach was successfully applied to 18 samples of $D$. odorifera. Large variation was observed for the contents of the 17 analytes. Sativanone and 3'-O-methylviolanone were the dominant compounds. The fragmentation behaviors of six flavonoids were investigated using UPLC with quadrupole time-of-flight tandem mass spectrometry. In negative ion electrospray ionization mass spectrometry, all the flavonoids yielded prominent $[\mathrm{M}-\mathrm{H}]^{-}$ions. Fragments for losses of $\mathrm{CH}_{3}, \mathrm{CO}$, and $\mathrm{CO}_{2}$ were observed in the mass spectra. Formononetin, liquiritigenin, isoliquiritigenin, sativanone, and alpinetin underwent retro-Diels-Alder fragmentations. The proposed method will be helpful for quality control of D. odorifera.
\end{abstract}

Keywords: Dalbergia odorifera; flavonoid; assay; fragmentation behavior; UPLC-MS/MS

\section{Introduction}

Dalbergia odorifera T. Chen (Leguminosae) is a semi-deciduous perennial tree that is indigenous to Hainan Province, South China. It has been introduced to and cultivated in Guangdong, Guangxi, Fujian, and Yunnan provinces, China [1]. Heartwood of D. odorifera is an important traditional Chinese medicine called "Jiangxiang" that is widely used to resolve stasis, stanch bleeding, regulate $q i$, and relieve pain [2]. In Korea, this heartwood is also used for treatment of blood stagnation, ischemia, swelling, necrosis, and rheumatic pain [3]. In addition, D. odorifera is commonly used as a component of commercial drug mixtures for cardiovascular treatment, including Guan-Xin-Er-Hao decoction [4], Qi-Shen-Yi-Qi decoction [5], Xinning tablets [2], and Tongxinluo capsules [6]. Also known 
as fragrant rosewood (Huanghuali in Chinese), D. odorifera is a valuable wood product for manufacture of furniture, artifacts, and musical instruments [7]. Due to its high medicinal and commercial value, many researchers have studied $D$. odorifera. The strong market demands combined with the slow growth of $D$. odorifera have resulted in production of counterfeit items. To ensure the safety and efficiency of $D$. odorifera in clinical practice, quantitation of its functional components is critical.

Phytochemical investigations have demonstrated that flavonoids and volatile oils are the main medicinal components of $D$. odorifera [8]. Flavonoids are secondary polyphenolic metabolites occurring commonly in many medicinal plants. Due to their extensive pharmacological activities, flavonoids are considered as the active principle components in many herbs. Recent investigations have shown that flavonoids in D. odorifera possess various biological activities, such as anti-inflammatory $[9,10]$, antioxidant [11], antitumor [12], antibacterial [13], and vasorelaxant activities [14]. Meanwhile, 3'-O-methylviolanone, sativanone, butein, liquiritigenin, butin, formononetin, etc. are the main compounds in D. odorifera [15-17]. Therefore, flavonoids could be considered as marker compounds to assess the quality of $D$. odorifera. However, no quantitative markers, except the content of its essential oils, have been selected for quality control of $D$. odorifera in the Chinese Pharmacopeia, which severely limits its clinical application and in-depth study. Among the analytical methods used for determination of flavonoids, the most widely used are based on high-performance liquid chromatography (HPLC) coupled with an ultraviolet (UV) or diode array detector [15-17]. The D. odorifera matrix is highly complex and the compounds of interest might be present in only minute quantities or accompanied by many other compounds with similar structures. In most cases, techniques like HPLC-UV will not be the optimum choice and can have long run times. A rapid, validated, and sensitive multi-component analytical method for quantification is required.

Ultra-high performance liquid chromatography (UHPLC) combined with triple quadrupole mass spectrometry (QqQ-MS) is considered one of the most efficient techniques for quantitative analysis, and can provide specific, sensitive, and selective quantitative results in multiple reaction monitoring (MRM) mode [18]. Although numerous UHPLC-tandem mass spectrometry (MS/MS) methods have been applied to the determination of bioactivities components in traditional Chinese medicines $[19,20]$, few studies have applied this method to quantitative analysis of flavonoids in D. odorifera [15-17,21]. Additionally, UHPLC-quadrupole time-of-flight (Q/TOF)-MS/MS has become increasingly important in compound identification because of its high selectivity, specificity, and accuracy [22].

In the present study, a rapid and sensitive UHPLC-QqQ-MS method was established using MRM mode for the simultaneous quantitative analysis of 17 flavonoids (daidzein, dalbergin, $3^{\prime}$-hydroxydaidein, liquiritigenin, isoliquiritigenin, alpinetin, butein, naringenin, butin, prunetin, eriodictyol, tectorigenin, pinocembrin, formononetin, genistein, sativanone, and $3^{\prime}$-O-methylviolanone, Figure 1) in D. odorifera grown in different areas of China. The fragmentation behaviors of six different types of flavonoids were explored using UHPLC-Q/TOF-MS/MS in negative ion mode. This study is an example of comprehensive quality control and expands the knowledge of quantitative and qualitative analysis of multiple flavonoids in D. odorifera.

\section{Results and Discussion}

\subsection{Method Development}

To develop a sensitive and accurate quantitative method, the analytes and internal standard (IS) were separately infused into the instrument to optimize the mass conditions. MS spectra were investigated in both positive and negative modes. All analytes showed maximum sensitivity in negative ion mode. For optimization of the MRM conditions, the cone voltage and collision voltage were optimized to acquire the richest relative abundance of precursor and daughter ions. Two transitions were monitored for identification of each component, and the transition with the higher intensity was selected for quantification. The retention time and MS parameters for each analyte are presented in Table 1. 

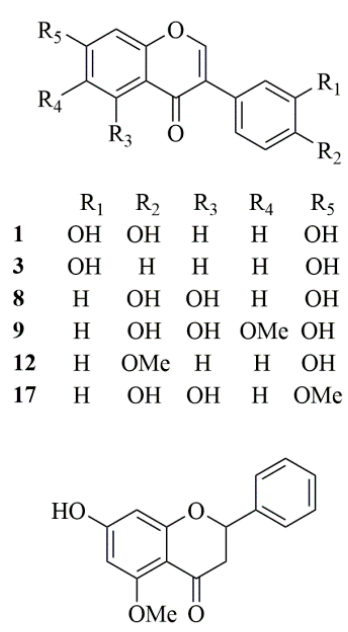

10

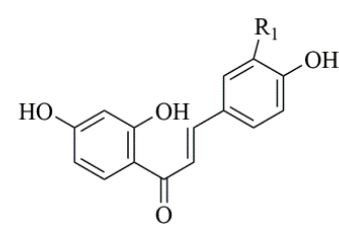

$\mathrm{R}_{1}$

$2 \mathrm{OH}$

$11 \mathrm{H}$

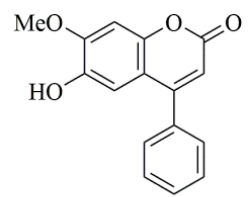

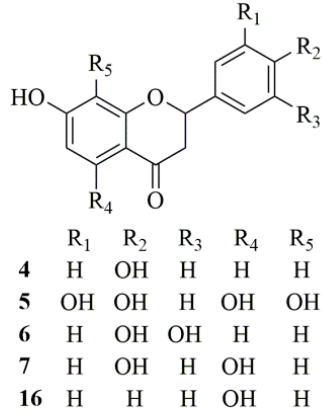

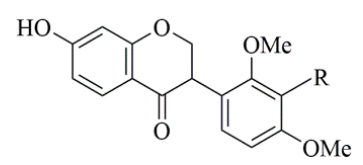

$14 \mathrm{R}=\mathrm{OMe} ; 15 \mathrm{R}=\mathrm{H}$

Figure 1. Chemical structures of the seventeen target compounds. 1, 3'-Hydroxydaidein; 2, Butein; 3, Daidzein; 4, Liquiritigenin; 5, Eriodictyol; 6, Butin; 7, Naringenin; 8, Genistein; 9, Tectorigenin; 10, Alpinetin; 11, Isoliquiritigenin; 12, Formononetin; 13, Dalbergin; 14, 3'-O-methylviolanone; 15 , Sativanone; 16, Pinocembrin; 17, Prunetin. Analytes numbers in the test is the same as in this figure.

To optimize the chromatographic behavior, the UHPLC conditions were explored. First, a Waters Acquity BEH C18 column $(100 \mathrm{~mm} \times 2.1 \mathrm{~mm}, 1.7 \mathrm{~mm})$ and Waters Acquity HSS T3 $(100 \mathrm{~mm} \times 2.1 \mathrm{~mm}, 1.8 \mu \mathrm{m})$ were examined. The Waters Acquity HSS T3 was chosen as it gave better separation and sharper peaks. Next, acetonitrile-water, methanol-water, and various additives (i.e., formic acid and acetic acid) were tested as potential mobile phases. Compared with the methanol-water system, the acetonitrile-water system gave better peak shapes and resolutions. For the modifiers, we found that acetic acid markedly inhibited the responses of the compounds. In addition, ionization of the flavonoids was inhibited if the concentration of formic acid was too high. Therefore, the concentration of formic acid was set at $0.05 \%$. The effects of the column temperature, flow rate, and elution procedure were also investigated. Finally, acetonitrile containing $0.05 \%$ formic acid with a flow rate of $0.25 \mathrm{~mL} / \mathrm{min}$ and the $40{ }^{\circ} \mathrm{C}$ column temperature were selected to achieve satisfactory separation in a short time (Figure 2).

\subsection{Optimization of the Extraction Conditions}

Sample preparation methods are of key importance in the analysis of samples with complex matrices, and especially in the simultaneous analysis of multiple compounds. To develop an efficient and appropriate extraction method for the 17 target components for UHPLC-MS/MS analysis, reflux extraction and ultrasonic extraction were compared used sample S9 $(0.2 \mathrm{~g})$. The two extraction methods gave similar results but the ultrasonic extraction was more convenient (Figure S1). Thus, ultrasonic extraction was chosen for subsequent experiments. To optimize the extraction, the extraction solvent $(50 \%, 60 \%, 70 \%$, or $80 \%$ methanol, $v / v)$, extraction volume $(15,20,25$, or $30 \mathrm{~mL})$, and extraction time $(30,45$, or $60 \mathrm{~min})$ were investigated. When the methanol concentration was increased from $50-70 \%$, the extraction efficiencies for the analytes increased (Figure 3A). However, when the methanol concentration was increased beyond $70 \%$, the extraction efficiencies showed no large increases. Therefore, we chose $70 \%$ methanol as the extraction solvent. There were no obvious differences in the contents of analytes between extraction volumes of 25 and $30 \mathrm{~mL}$ (Figure 3B), and the contents of some compounds some compounds (e.g., eriodictyol, naringenin, 3'-O-methylviolanone, sativanone, and pinocembrin) were higher than $20 \mathrm{~mL}$. The best extraction time for all components was $45 \mathrm{~min}$ (Figure 3C). Hence, the optimum conditions for extraction of D. odorifera were $0.2 \mathrm{~g}$ of dried sample, $25 \mathrm{~mL}$ of $70 \%$ methanol, and ultrasonic extraction for $45 \mathrm{~min}$. 


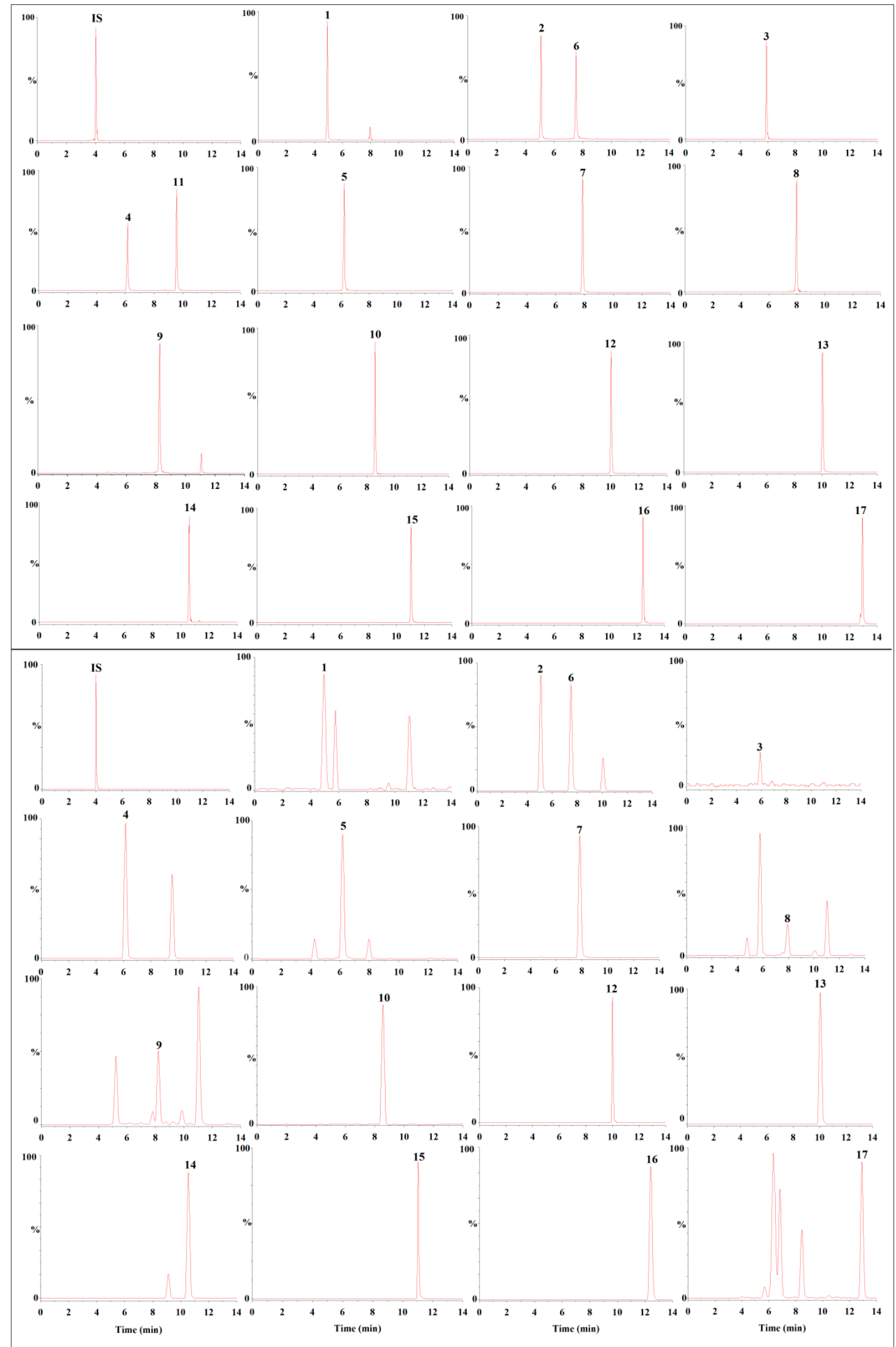

Figure 2. Ultra-high performance liquid chromatography (UHPLC)-MS/MS multiple reaction mode chromatograms of mixed standards (upper) and sample (lower, S9). 
Table 1. MS/MS parameters for 17 target compounds.

\begin{tabular}{|c|c|c|c|c|c|c|c|c|c|}
\hline No. & Compounds & Ion Mode & RT (min) & Precursor Ion & Cone Voltage (V) & Product ion $1^{Q}$ & $\begin{array}{c}\text { Collision } \\
\text { Energy (eV) }\end{array}$ & Product Ion $2^{\text {I }}$ & $\begin{array}{l}\text { Collision } \\
\text { Energy }(\mathrm{eV})\end{array}$ \\
\hline 1 & 3'-Hydroxydaidein & $\mathrm{ESI}^{-}$ & 4.97 & 269 & 52 & 213 & 28 & 241 & 24 \\
\hline 2 & Butein & $\mathrm{ESI}^{-}$ & 5.12 & 271 & 35 & 135 & 20 & 253 & 17 \\
\hline 3 & Daidzein & $\mathrm{ESI}^{-}$ & 5.95 & 253 & 51 & 208 & 31 & 224 & 26 \\
\hline 4 & Liquiritigenin & $\mathrm{ESI}^{-}$ & 6.23 & 255 & 40 & 135 & 15 & 119 & 22 \\
\hline 5 & Eriodictyol & $\mathrm{ESI}^{-}$ & 6.24 & 287 & 38 & 151 & 17 & 135 & 24 \\
\hline 6 & Butin & $\mathrm{ESI}^{-}$ & 7.59 & 271 & 39 & 135 & 31 & 91 & 37 \\
\hline 7 & Naringenin & $\mathrm{ESI}^{-}$ & 7.94 & 271 & 40 & 151 & 20 & 119 & 24 \\
\hline 8 & Genistein & $\mathrm{ESI}^{-}$ & 8.04 & 269. & 48 & 133 & 32 & 181 & 28 \\
\hline 9 & Tectorigenin & $\mathrm{ESI}^{-}$ & 8.30 & 2989 & 40 & 284 & 19 & 240 & 22 \\
\hline 10 & Alpinetin & $\mathrm{ESI}^{-}$ & 8.65 & 269 & 44 & 165 & 20 & 227 & 21 \\
\hline 11 & Isoliquiritigenin & $\mathrm{ESI}^{-}$ & 9.62 & 255 & 35 & 135 & 15 & 119 & 23 \\
\hline 12 & Formononetin & $\mathrm{ESI}^{-}$ & 10.08 & 267 & 45 & 252 & 22 & 223 & 25 \\
\hline 13 & Dalbergin & $\mathrm{ESI}^{-}$ & 10.09 & 267 & 38 & 180 & 27 & 252 & 18 \\
\hline 14 & 3'-O-methylviolanone & $\mathrm{ESI}^{-}$ & 10.62 & 329 & 46 & 135 & 38 & 299 & 36 \\
\hline 15 & Sativanone & $\mathrm{ESI}^{-}$ & 11.10 & 299 & 46 & 135 & 37 & 269 & 32 \\
\hline 16 & Pinocembrin & $\mathrm{ESI}^{-}$ & 12.47 & 255 & 42 & 107 & 25 & 171 & 25 \\
\hline 17 & Prunetin & $\mathrm{ESI}^{-}$ & 12.97 & 283 & 45 & 268 & 21 & 239 & 26 \\
\hline 18 & Rutin (IS) & $\mathrm{ESI}^{-}$ & 4.05 & 609 & 62 & 300 & 52 & 271 & 50 \\
\hline
\end{tabular}

Q: transitions for quantification; ${ }^{\text {I: }}$ transitions for identification. 

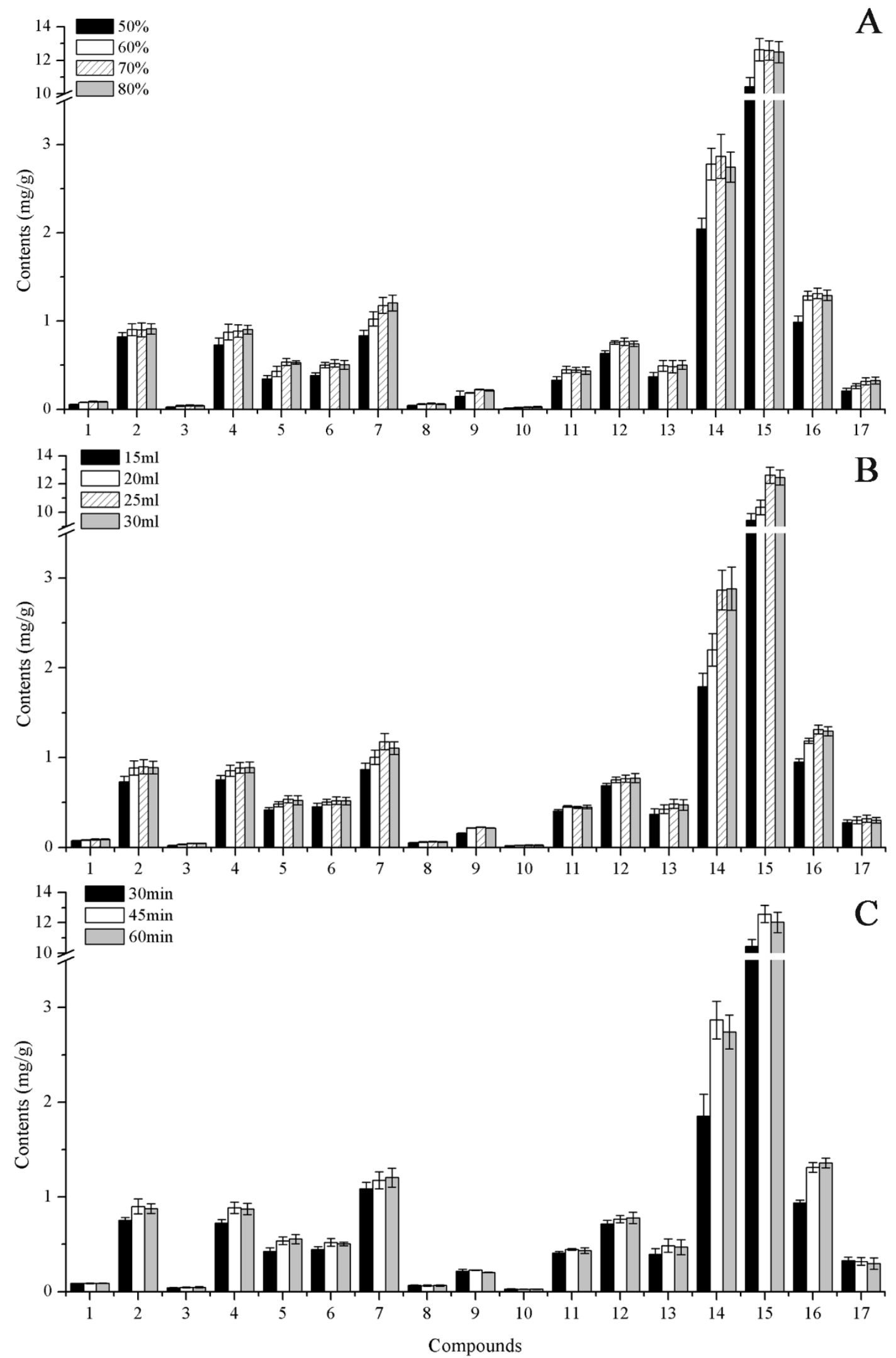

Figure 3. Effects of (A) solvent concentration, (B) solvent volume, and (C) extraction time on the extraction efficiency of target analytes in S9 sample. 


\subsection{Method Validation}

The developed UHPLC-MS/MS method for quantitation of 17 flavonoids was validated to determine the specificity, linearity, limit of detection (LOD), limit of quantification (LOQ), intra- and inter-day precisions, stability, and accuracy according to International Conference on Harmonization (ICH) guidelines for validation of analytical procedures [23].

\subsubsection{Specificity}

The representative MRM chromatograms of the mixed standard solution and real sample solution are shown in Figure 2. All of the target compounds could be distinguished using their retention times and precursor-to-product ion transitions. This indicates that the assay for D. odorifera is highly specific and selective.

\subsubsection{Linear range, LOD, and LOQ}

Linearity was evaluated using the coefficients of correlation $\left(r^{2}\right)$, which are listed along with the calibration curve equations, linear ranges, LOD, and LOQ in Table 2. Within the investigated concentration ranges, all compounds showed good linearity with $r^{2}$ ranging from 0.9986 to 0.9999 . The LOD and LOQ for each analyte were calculated using signal-to-noise ratios of three and ten, respectively. The LOD range was $0.085-6.080 \mathrm{ng} / \mathrm{mL}$ and the LOQ range was $0.256-18.840 \mathrm{ng} / \mathrm{mL}$ for the 17 target compounds, which showed the method had high sensitivity.

\subsubsection{Precision, Repeatability, and Stability}

The intra- and inter-day variability were measured to assess the precision of the developed method using sample 9. The intra-day precision was evaluated by analyzing six replicates prepared from sample 9, and the inter-day precision was examined over three consecutive days with samples per day. The repeatability was determined by injection of six samples prepared following the same procedure (Section 2.4). The stability of the sample solution over $24 \mathrm{~h}$ at room temperature was also evaluated. For the precision, repeatability, and stability tests, the percent relative standard deviations were within $5.0 \%$ (Table 2 ).

\subsubsection{Accuracy}

To further evaluate the accuracy of the proposed method, a recovery test was carried out by spiking three levels (80\%, 100\%, and 120\% of the known amount) of mixture standard solution and corresponding IS standards to known amount samples. Next, the spiked samples were extracted and analyzed using the proposed method, and then, triplicate experiments were performed at each level. The recoveries were calculated using the following equation: Recovery $(\%)=$ (total amount detected amount in original sample)/amount spiked $\times 100 \%$. The recovery for each compound was in the range of $94.18-101.97 \%$ and the relative standard deviation was less than $6.0 \%$ (Table S1 in Supplementary Materials). The results implied that the developed UHPLC-MS/MS was precise, accurate, sensitive, and reliable enough for simultaneous quantitative analysis of the 17 target compounds in $D$. odorifera. 
Table 2. Curves, test range, limit of detection (LOD), limit of quantification (LOQ), precision, and repeatability for the seventeen analytes.

\begin{tabular}{|c|c|c|c|c|c|c|c|c|c|}
\hline \multirow{2}{*}{ No. } & \multirow{2}{*}{ Compounds } & \multirow{2}{*}{ Calibration Curves } & \multirow{2}{*}{$r^{2}$} & \multirow{2}{*}{ Linear Range (ng/mL) } & \multirow{2}{*}{ LOQ (ng/mL) } & \multirow{2}{*}{ LOD (ng/mL) } & \multicolumn{2}{|c|}{ Precision (RSD, \%) } & \multirow{2}{*}{$\begin{array}{c}\text { Repeatability } \\
(\mathrm{RSD}, \%, n=6)\end{array}$} \\
\hline & & & & & & & Intra-Day & Inter-Day & \\
\hline 1 & 3'-Hydroxydaidein & $Y=0.934 X-0.0436$ & 0.9991 & $5.40-1350$ & 5.400 & 1.600 & 2.43 & 3.23 & 3.45 \\
\hline 2 & Butein & $Y=0.1675 X-0.198$ & 0.9993 & $1.41-2820$ & 1.410 & 0.470 & 2.49 & 4.85 & 4.53 \\
\hline 3 & Daidzein & $Y=0.9697 X-0.061$ & 0.9989 & $3.02-1510$ & 3.020 & 1.000 & 1.74 & 3.01 & 2.98 \\
\hline 4 & Liquiritigenin & $Y=0.1813 X+0.0075$ & 0.9999 & $1.61-3220$ & 1.610 & 0.500 & 1.25 & 2.26 & 3.18 \\
\hline 5 & Eriodictyol & $Y=0.1802 X-0.0166$ & 0.9997 & $1.36-1360$ & 1.360 & 0.453 & 2.38 & 2.45 & 2.06 \\
\hline 6 & Butin & $Y=0.1163 X-0.0568$ & 0.9986 & $1.51-3020$ & 1.510 & 0.458 & 1.85 & 3.54 & 2.77 \\
\hline 7 & Naringenin & $Y=0.2291 X-0.0722$ & 0.9989 & $2.72-1360$ & 2.720 & 0.906 & 2.07 & 4.61 & 4.06 \\
\hline 8 & Genistein & $Y=0.8139 X-0.2152$ & 0.9988 & $3.82-1910$ & 3.820 & 1.528 & 0.76 & 3.33 & 2.54 \\
\hline 9 & Tectorigenin & $Y=0.203 X-0.2161$ & 0.9987 & $2.44-1220$ & 2.440 & 0.813 & 1.96 & 2.40 & 1.95 \\
\hline 10 & Alpinetin & $Y=0.5127 X-0.0544$ & 0.9996 & $5.36-1340$ & 5.360 & 1.790 & 2.85 & 4.94 & 4.76 \\
\hline 11 & Isoliquiritigenin & $Y=0.1308 X+0.0284$ & 0.9996 & $1.416-1770$ & 1.416 & 0.480 & 0.45 & 3.02 & 3.67 \\
\hline 12 & Formononetin & $Y=0.0516 X-0.0608$ & 0.9993 & $0.516-1290$ & 0.516 & 0.172 & 1.78 & 1.90 & 3.32 \\
\hline 13 & Dalbergin & $Y=0.2867 X-0.0665$ & 0.9991 & $0.256-1280$ & 0.256 & 0.085 & 3.51 & 4.85 & 4.61 \\
\hline 14 & 3'-O-methylviolanone & $Y=0.6244 X+0.0119$ & 0.9989 & $9.90-2970$ & 9.900 & 3.300 & 2.08 & 4.49 & 2.87 \\
\hline 15 & Sativanone & $Y=0.675 X-0.1047$ & 0.9991 & $18.84-5652$ & 18.840 & 6.080 & 1.24 & 1.26 & 4.73 \\
\hline 16 & Pinocembrin & $Y=0.3485 X-0.0569$ & 0.9992 & $2.66-1330$ & 2.660 & 0.870 & 1.04 & 1.94 & 3.82 \\
\hline 17 & Prunetin & $Y=0.0489 X-0.0657$ & 0.9989 & $1.12-2240$ & 1.120 & 0.374 & 1.91 & 3.34 & 3.61 \\
\hline
\end{tabular}




\subsection{Method Application}

The validated method was applied to determine the 17 selected flavonoids in 18 samples of D. odorifera. Representative MRM chromatograms are shown in Figure 2 and the quantitative results are shown in Table 3. The contents of the 17 analytes varied in different batches of $D$. odorifera. Sativanone and 3'-O-methylviolanone were the dominant compounds in D. odorifera. The content of sativanone in all batches ranged from 5.8806 to $24.1200 \mathrm{mg} / \mathrm{g}$ (4.10-fold variation), and that of $3^{\prime}$-O-methylviolanone ranged from 0.6973 to $7.583 \mathrm{mg} / \mathrm{g}$ (10.87-fold variation). The content of daidzein in most samples was lower than the LOQ. For $3^{\prime}$-hydroxydaidein, genistein, and alpinetin, the contents were also relatively low $(<0.2 \mathrm{mg} / \mathrm{g})$. The trends observed in our results were similar to those in previous studies [15-17]. For example, Liu et al. [15] analyzed 10 major flavonoids in D. odorifera by HPLC-UV and found that sativanone $(1.45-20.90 \mathrm{mg} / \mathrm{g})$ was dominant. The average contents of other flavonoids (e.g., liquiritigenin, formononetin, and dalbergin) were higher than our results. In another study, seven flavonoids were analyzed in D. odorifera by Li et al. [17] and the obtained concentration ranges for the detected analytes (liquiritigenin, formononetin, isoliquiritigenin, and naringenin) were similar to those in the present study. Variation in the levels of flavonoids among the samples could be caused by differences in geographical conditions, the tree ages, plant origins, and storage conditions. The results suggest that UHPLC-MS/MS is a very powerful technique for quantitative analysis of multiple components of $D$. odorifera because it is rapid and sensitive.

\subsection{Fragmentation Pathways Analysis}

To date, 99 flavonoids have been isolated from D. odorifera [21]. These compounds have the same basic skeleton with different substituents. A total of 17 flavonoids, including six isoflavones ( $3^{\prime}$-hydroxydaidein, daidzein, genistein, tectorigenin, formononetin, and prunetin), five flavanones (liquiritigenin, eriodictyol, butin, naringenin, and pinocembrin), two chalcones (butein and isoliquiritigenin), two isoflavanones (sativanone and $3^{\prime}$-O-methylviolanone), one flavone (alpinetin), and one neoflavone (dalbergin) were quantified in the present study. Negative ion electrospray ionization (ESI) mode was found to be more sensitive than positive ion mode for detecting flavonoids. To further identify the compounds in D. odorifera, fragmentation pathways of six representative flavonoids (formononetin, pinocembrin, isoliquiritigenin, sativanone, alpinetin, and dalbergin) of $D$. odorifera were examined by UPLC-Q/TOF-MS/MS in negative ionization mode.

Formononetin is a methoxylated isoflavone. The suggested fragmentation pathway of formononetin is shown in Figure 4a. The main and typical fragmentation ions of this compound result from successive or simultaneous losses of $\mathrm{CH}_{3}, \mathrm{CHO}, \mathrm{CO}$, and $\mathrm{CO}_{2}$, which are attributed to the $4^{\prime}-\mathrm{OCH}_{3}$ isoflavone type [24]. The base peak ion of formononetin at $m / z 252.0491\left[\mathrm{M}-\mathrm{H}_{-} \mathrm{CH}_{3}\right]^{-}$is formed by loss of a $\mathrm{CH}_{3}$ group. This result is consistent with a previous study that showed that loss of $\mathrm{CH}_{3}$ was characteristic of fragmentation in methoxylated flavonoids [25]. As the collision energy increased, abundant characteristic fragment ions were observed at $m / z 223.0437\left[\mathrm{M}-\mathrm{H}_{-} \mathrm{CH}_{3}-\mathrm{CHO}^{-}, \mathrm{m} / z 224.0480\right.$ $\left[\mathrm{M}-\mathrm{H}-\mathrm{CH}_{3}-\mathrm{CO}^{-}, \mathrm{m} / \mathrm{z} 208.0563\left[\mathrm{M}-\mathrm{H}-\mathrm{CH}_{3}-\mathrm{CO}_{2}\right]^{-}, \mathrm{m} / \mathrm{z} \text { 195.0491 [M - H-CH }-\mathrm{CHO}_{3}-\mathrm{CO}\right]^{-}, \mathrm{m} / \mathrm{z}$ $180.0627\left[\mathrm{M}-\mathrm{H}-\mathrm{CH}_{3}-\mathrm{CO}_{2}-\mathrm{CO}\right]^{-}$, and $m / z 167.0543\left[\mathrm{M}-\mathrm{H}_{-} \mathrm{CH}_{3}-\mathrm{CHO}-2 \mathrm{CO}\right]^{-}$. It is worth mentioning that neutral loss of $\mathrm{CO}_{2}$ is common for isoflavones in MS/MS and the fragment ion at $\mathrm{m} / \mathrm{z} 223.0437$ differed from $\mathrm{m} / \mathrm{z} 267.0666$ by $44 \mathrm{Da}$, which is typically assigned as neutral loss of $\mathrm{CO}_{2}$. However, the TOF/MS revealed that the formula of $m / z 223.0437$ was $\mathrm{C}_{14} \mathrm{H}_{7} \mathrm{O}_{3}$, and this was formed by loss of $\mathrm{C}_{2} \mathrm{H}_{4} \mathrm{O}$ rather than $\mathrm{CO}_{2}$. Therefore, the typical loss of $\mathrm{CO}_{2}$ did not occur in this case. Instead, this fragment was produced via losses of $\mathrm{CH}_{3}$ and $\mathrm{CHO}$ at the $4^{\prime}$-position [26]. Fragment ions at $\mathrm{m} / \mathrm{z}$ $132.0259\left[{ }^{1,3} \mathrm{~B}-\mathrm{H}\right]^{-}$and $135.0125\left[^{1,3} \mathrm{~A}-\mathrm{H}\right]^{-}$were produced by retro-Diels-Alder (RDA) fragmentation in the C-ring of formononetin. 
Table 3. Contents of 17 analytes in 18 batches of samples $(\mathrm{mg} / \mathrm{g})$.

\begin{tabular}{|c|c|c|c|c|c|c|c|c|c|c|c|c|c|c|c|c|c|}
\hline & 1 & 2 & 3 & 4 & 5 & 6 & 7 & 8 & 9 & 10 & 11 & 12 & 13 & 14 & 15 & 16 & 17 \\
\hline S1 & 0.1004 & 0.0769 & 0.0546 & 0.7228 & $<$ LOQ & 1.7084 & 0.3902 & 0.0396 & 0.9048 & ND & 0.2994 & 1.1047 & 0.0255 & 0.6973 & 5.8806 & 0.1901 & 0.1207 \\
\hline S2 & 0.1052 & 0.3714 & 0.0422 & 0.8499 & 0.0424 & 0.2862 & 0.3362 & 0.0302 & 0.7439 & ND & 0.4019 & 1.0490 & 0.0452 & 1.4732 & 6.5233 & 0.2467 & 0.1119 \\
\hline S3 & 0.0232 & 0.4628 & $<\mathrm{LOQ}$ & 0.6125 & 0.3140 & 0.2346 & 0.6570 & 0.0379 & 0.2066 & 0.0436 & 0.2881 & 0.2712 & 0.1724 & 1.8881 & 8.7726 & 0.8209 & 0.1794 \\
\hline S4 & 0.1340 & 0.0865 & 0.1102 & 1.0619 & $<\mathrm{LOQ}$ & 0.0400 & 0.3603 & 0.0828 & 0.8650 & 0.0860 & 0.5860 & 1.2523 & 0.0171 & 0.8051 & 9.5705 & 0.2324 & 0.1228 \\
\hline S5 & $<$ LOQ & 0.4860 & $<$ LOQ & 0.1908 & 0.5322 & 0.2570 & 0.8737 & 0.0234 & 0.1184 & 0.0141 & 0.1192 & 0.0667 & 0.0302 & 7.5830 & 18.2890 & 0.2874 & 0.0806 \\
\hline S6 & 0.0281 & 0.9359 & $<\mathrm{LOQ}$ & 0.3571 & 0.5365 & 0.5594 & 0.5046 & 0.0226 & 0.1325 & 0.0241 & 0.1852 & 0.3650 & 0.0164 & 3.8599 & 24.1200 & 0.3118 & 0.1195 \\
\hline S7 & $<\mathrm{LOQ}$ & 0.4820 & $<$ LOQ & 0.2767 & 0.0364 & 0.2680 & 0.1307 & 0.0068 & 0.0751 & 0.0310 & 0.1744 & 0.1930 & 0.0701 & 1.0216 & 7.2718 & 0.0500 & 0.0147 \\
\hline S8 & 0.0366 & 0.7531 & $<$ LOQ & 0.5434 & 0.0658 & 0.5064 & 0.2038 & 0.0251 & 0.1711 & 0.0665 & 0.3136 & 0.3630 & 0.1084 & 1.4806 & 7.3738 & 0.1676 & 0.0381 \\
\hline S9 & 0.0888 & 0.9047 & 0.0442 & 0.8766 & 0.5470 & 0.5149 & 1.1659 & 0.0688 & 0.2205 & 0.0258 & 0.4645 & 0.7756 & 0.4961 & 2.8056 & 12.6911 & 1.3608 & 0.3284 \\
\hline S10 & 0.0423 & 0.8399 & $<$ LOQ & 0.5717 & 0.4619 & 0.5250 & 0.7914 & 0.0393 & 0.3628 & 0.0239 & 0.3086 & 0.6162 & 0.3954 & 3.5777 & 14.6963 & 1.1722 & 0.2915 \\
\hline S11 & 0.0961 & 0.9512 & 0.0380 & 1.0640 & 0.0907 & 0.6402 & 0.2374 & 0.0079 & 0.2600 & 0.0130 & 0.5423 & 0.7814 & 0.4026 & 1.6566 & 6.7257 & 0.4049 & 0.0701 \\
\hline S12 & 0.0375 & 0.6593 & $<\mathrm{LOQ}$ & 1.0338 & 0.7855 & 0.4597 & 1.5180 & 0.0820 & 0.4318 & 0.0226 & 0.6463 & 0.4233 & 0.0024 & 3.3384 & 15.1491 & 1.2503 & 0.4409 \\
\hline S13 & 0.0431 & 1.0001 & $<\mathrm{LOQ}$ & 0.8340 & 1.1740 & 0.6573 & 2.4237 & 0.1641 & 0.2210 & 0.0375 & 0.4746 & 0.6412 & 0.1302 & 4.5177 & 23.7313 & 2.1126 & 0.5725 \\
\hline S14 & 0.0625 & 1.9857 & $<$ LOQ & 1.8790 & 0.0830 & 1.6506 & 0.1734 & ND & 0.2656 & 0.0111 & 1.3703 & 1.1935 & 0.4145 & 1.0923 & 8.3085 & 0.1969 & 0.0464 \\
\hline S15 & 0.0621 & 0.9035 & $<$ LOQ & 0.5811 & 0.2317 & 0.4897 & 0.7012 & 0.0414 & 0.2702 & 0.0211 & 0.3043 & 0.6900 & 0.1995 & 2.5788 & 15.1260 & 0.5150 & 0.2174 \\
\hline S16 & 0.0536 & 0.5246 & 0.0399 & 0.4060 & 0.0321 & 0.2395 & 0.1328 & 0.0161 & 0.1031 & 0.0131 & 0.2361 & 0.3781 & 0.1192 & 0.8817 & 6.6720 & 0.1057 & 0.0315 \\
\hline S17 & $<\mathrm{LOQ}$ & 0.2551 & 0.0544 & 0.6073 & $<$ LOQ & 0.1680 & 0.3325 & 0.0300 & 0.1935 & 0.0161 & 0.3011 & 0.4070 & 0.0034 & 1.5828 & 21.6877 & 0.3601 & 0.0606 \\
\hline S18 & ND & 0.1246 & ND & 0.1123 & 0.0243 & 0.0460 & 0.1787 & 0.0053 & 0.0580 & 0.0869 & 0.0719 & 0.0731 & 0.0252 & 1.1494 & 8.3910 & 0.1419 & 0.0325 \\
\hline
\end{tabular}


a
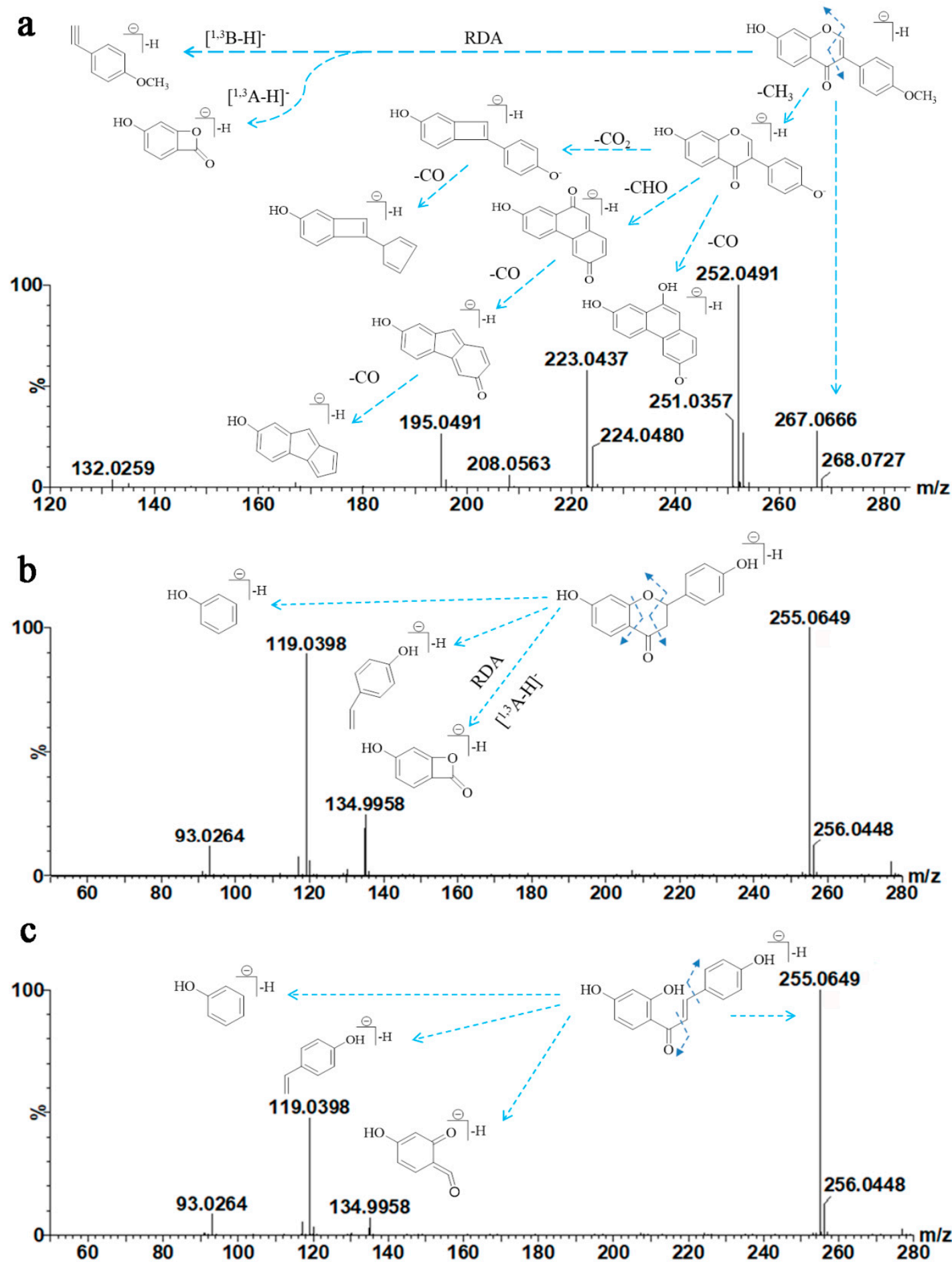

Figure 4. MS/MS spectra and the proposed fragmentation pathway of formononetin (a), liquiritigenin (b), and isoliquiritigenin (c).

Liquiritigenin gave a precursor ion $[\mathrm{M}-\mathrm{H}]^{-}$at $m / z 255.0649$ (Figure $4 b$ ). In the MS/MS spectrum, characteristic ions were observed at $m / z$ 134.9958, 119.0398 and 93.0264, which were consistent with the typical $\left[{ }^{1,3} \mathrm{~A}-\mathrm{H}\right]^{-}$and $\left[{ }^{1,3} \mathrm{~B}-\mathrm{H}\right]^{-}$fragments. Isoliquiritigenin showed similar fragmentation behavior to liquiritigenin (Figure $4 \mathrm{c}$ ).

The MS/MS spectra and fragmentation pathway of sativanone are shown in Figure 5a. Generally, losses of $\mathrm{CH}_{3}$ and $\mathrm{CO}_{2}$ were prominent. Loss of $\mathrm{CH}_{3}$ from the B-ring of sativanone yielded fragments at $m / z 284.0719\left[\mathrm{M}-\mathrm{H}-\mathrm{CH}_{3}\right]$ and $m / z 269.0158\left[\mathrm{M}-\mathrm{H}-\mathrm{CH}_{3}\right]$ produced from the precursor ion at 
$299.0566\left([\mathrm{M}-\mathrm{H}]^{-}\right)$. Loss of $\mathrm{CO}_{2}$ from $\mathrm{m} / z 269.0158$ yielded $\left[\mathrm{M}-\mathrm{H}-2 \mathrm{CH}_{3}-\mathrm{CO}_{2}\right]^{-}(\mathrm{m} / \mathrm{z} 225.1346)$. A fragment ion at $m / z 134.9958\left[{ }^{1,3} \mathrm{~A}-\mathrm{H}\right]^{-}$was generated after RDA cracking, and further loss of $\mathrm{CO}_{2}$ from $\mathrm{m} / \mathrm{z} 134.9958$ produced $\left[{ }^{1,3} \mathrm{~A}-\mathrm{H}-\mathrm{CO}_{2}\right]^{-}$at $\mathrm{m} / \mathrm{z}$ 91.0106. Additionally, fragmentation at the C-ring produced a ${ }^{0,3} \mathrm{~B}^{-}$ion at $m / z 179.0582$ with low abundance. Further loss of one $\mathrm{H}_{2} \mathrm{O}$ produced an ion at $m / z$ 161.0083. This fragmentation pathway was consistent with the previous report of Zhao et al. [27].

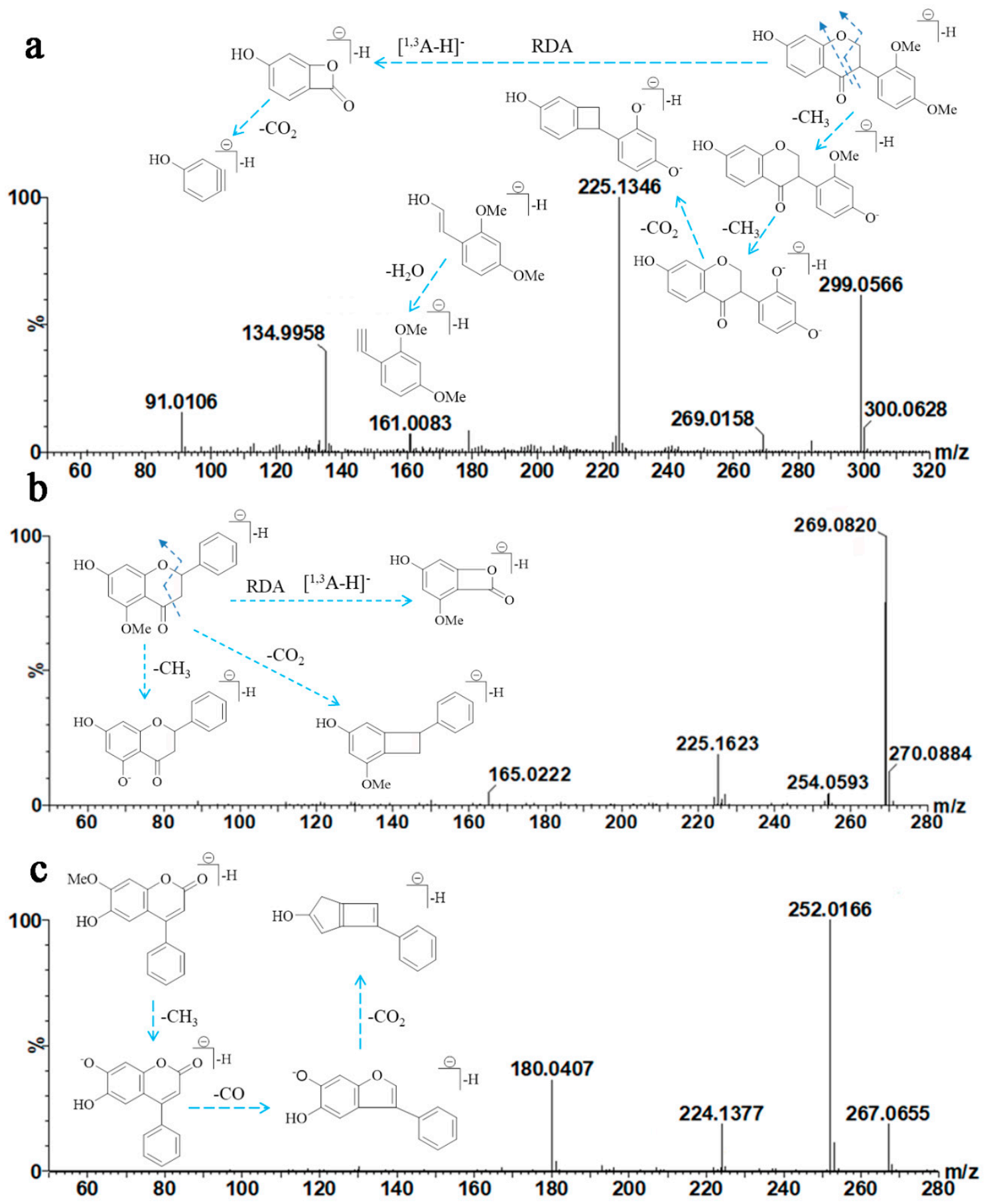

Figure 5. MS/MS spectra and the proposed fragmentation pathway of sativanone (a), alpinetin (b), and dalbergin (c).

The fragmentation behavior for alpinetin is shown in Figure 5b. Alpinetin gave a $[\mathrm{M}-\mathrm{H}]^{-}$ion at $m / z 269.0820$ as the base peak. Two radical anions at $m / z 254.0539\left[\mathrm{M}-\mathrm{H}-\mathrm{CH}_{3}\right]^{-}$and $225.1623[\mathrm{M}$ 
- $\left.\mathrm{H}-\mathrm{CO}_{2}\right]^{-}$formed by loss of $\mathrm{CH}_{3}$ and $\mathrm{CO}_{2}$ from the precursor anion. Additionally, a peak at $\mathrm{m} / \mathrm{z}$ 165.0222 $\left[{ }^{1,3} \mathrm{~A}-\mathrm{H}\right]^{-}$was observed after RDA fragmentation.

Little research has been conducted on the fragmentation pathways of neoflavones [28]. The mass spectrum of dalbergin (Figure 5c) exhibited significant ions at $\mathrm{m} / \mathrm{z} 267.0655,252.0166,224.1377$, and 180.0407. The precursor ion lost one $\mathrm{CH}_{3}$ to give an ion at $\mathrm{m} / \mathrm{z} 252.0166$. Subsequent loss of $\mathrm{CO}$ from this ion generated a fragment ion $\mathrm{m} / \mathrm{z}$ 224.1377. Further loss of $\mathrm{CO}_{2}$ from $\mathrm{m} / \mathrm{z} 224.1377$ yielded a fragment ion at $m / z 180.0407$.

In negative ion ESI-MS/MS, all target analytes yielded prominent $[\mathrm{M}-\mathrm{H}]^{-}$ions. Some common features, such as loss of $\mathrm{CH}_{3}, \mathrm{CO}$, and $\mathrm{CO}_{2}$, were observed in the MS/MS spectra, and were consistent with the literature. The $\left[\mathrm{M}-\mathrm{H}-\mathrm{CH}_{3}\right]^{-}$ion was a characteristic fragment of methoxylated flavonoids (formononetin, alpinetin, and dalbergin). In addition, $\left[\mathrm{M}-\mathrm{H}-2 \mathrm{CH}_{3}\right]^{-}$fragments were observed for dimethoxylated flavonoids (sativanone). Therefore, loss of one or two $\mathrm{CH}_{3}$ could be adopted to identify single- or multi-methoxylated flavonoids in negative ion ESI-MS/MS. Loss of CO and $\mathrm{CO}_{2}$ from $[\mathrm{M}-\mathrm{H}]^{-}$ions was attributed to the structure of the C-ring. The $[\mathrm{M}-\mathrm{H}]^{-}$ions of formononetin, liquiritigenin, isoliquiritigenin, sativanone, and alpinetin underwent RDA fragmentation. However, RDA fragmentation was not observed for dalbergin, which may be related to its specific structural characteristics.

\section{Materials and Methods}

\subsection{Solvents and Chemicals}

HPLC-grade acetonitrile and formic acid were purchased from Thermo Fisher Scientific (Fair Lawn, NJ, USA). Analytical grade methanol was purchased from Beijing Chemical Works (Beijing, China). Deionized water was prepared using a Milli-Q system (Millipore, Milford, MA, USA). Reference standards of daidzein, dalbergin, 3'-hydroxydaidein, liquiritigenin, isoliquiritigenin, alpinetin, butein, naringenin, butin, prunetin, eriodictyol, and tectorigenin were purchased from Chengdu Chroma-Biotechnology Co., Ltd. (Chengdu, China). Rutin for use as an internal standard (IS), pinocembrin, formononetin, and genistein were obtained from Sichuan Victory Biological Technology Co., Ltd. (Chengdu, China). The purities of all standards were above $98.0 \%$. We isolated sativanone and $3^{\prime}-O$-methylviolanone from the heartwood of $D$. odorifera $T$. Chen. The structures of these two compounds were unambiguously identified by NMR techniques, and their purities were determined to be above $96 \%$ by HPLC.

Heartwood samples of Dalbergia odorifera T. Chen $(n=18)$ were collected from different areas in China. The samples were identified by Prof. Jianhe Wei (Institute of Medicinal Plant Development, Chinese Academy of Medical Science \& Peking Union Medical College, Beijing, China), and voucher specimens (No. 469027-LD-020) were deposited in the Resource Center for Chinese Materia Media (Hainan Branch Institute of Medicinal Plant Development, Chinese Academy of Medical Sciences \& Peking Union Medical College, China).

\subsection{UHPLC-QqQ-MS/MS}

Analyses were performed on a UHPLC system (Acquity H-Class, Waters Corp., Milford, MA, USA) with a binary solvent manager, a column manager, and a sample manager. The sample was separated on a Waters Acquity HSS T3 column $(100 \mathrm{~mm} \times 2.1 \mathrm{~mm}, 1.8 \mu \mathrm{m}$; Waters Corp. $)$ and the column temperature was set at $40^{\circ} \mathrm{C}$. The mobile phase consisted of acetonitrile (A) and water containing $0.05 \%$ formic acid (B) with a flow rate of $0.25 \mathrm{~mL} / \mathrm{min}$. The following gradient program was used: $5-30 \%$ A from $0-2 \mathrm{~min}, 30-33 \%$ A from $2-5 \mathrm{~min}, 33-53 \%$ A from $5-13 \mathrm{~min}$, held at $53 \%$ A for $3 \mathrm{~min}, 53-95 \%$ A from $16-18 \mathrm{~min}$, held at $95 \%$ A for $2 \mathrm{~min}$, and $95-5 \%$ A from $20-22 \mathrm{~min}$. For UHPLC-MS/MS analysis, a Waters QqQ-MS (Xevo TQ-D, Waters Corp.) was connected to the Waters UHPLC instrument via an electrospray ionization (ESI) source. Analytes were quantified by MRM in negative ionization mode with argon as the collision gas. All MS parameters were optimized in 
the combined mode. The following ESI ion source parameters were used: capillary voltage, $2.5 \mathrm{kV}$; source temperature, $150{ }^{\circ} \mathrm{C}$; desolvation temperature, $400{ }^{\circ} \mathrm{C}$; cone gas (nitrogen) flow rate, $50 \mathrm{~L} / \mathrm{h}$; and desolvation gas (nitrogen) flow rate, $600 \mathrm{~L} / \mathrm{h}$. The UHPLC-MS/MS parameters, including the precursor-to-product ion transitions, cone voltage, and collision energy, are listed in Table 1.

\subsection{UHPLC-Q/TOF-MS/MS}

Flavonoid fragmentation was performed on a quadrupole time-of-flight mass spectrometer (Xevo G2-XS, Waters Corp.) equipped with an ESI source and coupled to the UPLC system. The above UHPLC conditions were used for UHPLC-Q/TOF-MS/MS. Detection was implemented in the MS ${ }^{\mathrm{E}}$ centroid mode over a mass range of 500-1000 Da with a scan rate of $10 \mathrm{Da} / \mathrm{s}$. The analyzer sensitivity mode was used. Leukine enkephalin was infused using LockSpray via a reference probe for in-run mass corrections. The ESI ion source parameters were as follows: capillary voltage, $2.5 \mathrm{kV}$; desolvation gas (nitrogen) flow rate, $600 \mathrm{~L} / \mathrm{h}$, desolvation temperature, $40{ }^{\circ} \mathrm{C}$; cone gas flow rate, $50 \mathrm{~L} / \mathrm{h}$; and source temperature, $150{ }^{\circ} \mathrm{C}$. The collision energy was ramped in a high energy function from 20 to $60 \mathrm{eV}$ using argon as the collision gas. MassLynx software (Waters Corp.) was used for post-acquisition analysis.

\subsection{Sample Preparation}

The materials were pulverized and dried to a constant mass before use. A $0.20 \mathrm{~g}$ sample was extracted with $25 \mathrm{~mL}$ of $70 \%$ methanol $(v / v)$ in an ultrasonic water bath for $45 \mathrm{~min}$ and then filtered. An aliquot $(1 \mathrm{~mL})$ of the filtrate was transferred into a 15-mL screw cap plastic tube containing $9 \mathrm{~mL}$ of $70 \%$ aqueous methanol and shaken immediately for $1 \mathrm{~min}$ using a vortex mixer. Then, $0.5 \mathrm{~mL}$ of IS $(1.0 \mu \mathrm{g} / \mathrm{mL})$ was added to $0.5 \mathrm{~mL}$ of the solution and the mixture was vortexed for $30 \mathrm{~s}$. The obtained solution was filtered through a $0.22-\mu \mathrm{m}$ micropore membrane before use. A $5 \mu \mathrm{L}$ sample was injected into the UHPLC instrument for analysis.

\subsection{Standard Solution Preparation}

Standard stock solutions of 17 reference standards with a concentration range of 24.4 to $113.04 \mu \mathrm{g} / \mathrm{mL}$ were separately prepared by dissolution in methanol. An initial stock solution was prepared as a mixture of the above stock solutions. A series of working solutions of the analytes were obtained by diluting the mixed stock solution with methanol to the appropriate concentration. Then, $0.5 \mathrm{~mL}$ of IS $(1.0 \mu \mathrm{g} / \mathrm{mL})$ was added to $0.5 \mathrm{~mL}$ of the working solutions and the mixture was vortexed for $30 \mathrm{~s}$. All of the solutions were stored at $4{ }^{\circ} \mathrm{C}$ and filtered through a $0.22-\mu \mathrm{m}$ nylon membrane before injection into the UHPLC system.

\section{Conclusions}

In the present study, a sensitive and rapid UHPLC-ESI-MS/MS method was established for the simultaneous quantitative analysis of 17 flavonoids in the heartwood of D. odorifera and successfully applied to 18 samples. Satisfactory validation parameters were obtained, including specificity, linearity, precision, accuracy, and stability, and the extraction method was optimized. Taking the contents of the target compounds into consideration, the quantification results indicated that the quality of D. odorifera varied. The MS fragmentation pathways of flavonoids discussed here could facilitate rapid screening and structural characterization of these compounds by LC-MS/MS. Our results suggest that UHPLC-MS/MS could be a useful tool for quality assessment of D. odorifera using flavonoids as markers.

Supplementary Materials: The following are available online, Table S1: Recoveries of 17 target compounds.

Author Contributions: Conceptualization, J.W. designed the experiments; X.Z. performed the experiments and wrote the paper; S.Z. and D.L. optimized the condition of samples pretreatment. M.Y. contributed in polishing the language and grammer. All authors have read and agreed to the published version of the manuscript. 
Funding: This research was funded by the Key Research Project of Hainan Province, China (Grant No. ZDYF2018123) and the CAMS Innovation Fund for Medical Sciences (CIFMS) (Grant No. 2016-I2M-2-003).

Conflicts of Interest: The authors declare that they have no conflict of interest.

\section{References}

1. Liu, X.J.; Xu, D.P.; Yang, Z.J.; Zhang, N.G. Geographic variations in seed germination of Dalbergia odorifera T. Chen in response to temperature. Ind. Crop. Prod. 2017, 102, 45-50. [CrossRef]

2. Chinese Pharmacopoeia Commission. Pharmacopoeia of People's Republic of China; Chemical Industry Press: Beijing, China, 2015.

3. Kang, T.H.; Tian, Y.H.; Kim, Y.C. Isoliquiritigenin: A competitive tyrosinase inhibitor from the heartwood of Dalbergia odorifera. J. Appl. Pharm. 2005, 13, 32-34.

4. Qin, F.; Liu, Y.X.; Zhao, X.; Huang, X.; Ren, P.; Zhu, Z.Y. Chinese medicinal formula Guan-Xin-Er-Hao protects the heart against oxidative stress induced by acute ischemic myocardial injury in rats. Phytomedicine 2009, 16, 215-221. [CrossRef] [PubMed]

5. Yu, J.H.; Zhang, W.; Zhang, Y.Q.; Wang, Y.D.; Zhang, B.L.; Fan, G.W.; Zhu, Y. A critical courier role of volatile oils from Dalbergia odorifera for cardiac protection in vivo by QiShenYiQi. Sci. Rep. 2017, 7, 7353. [CrossRef] [PubMed]

6. Zhang, N.; Gao, Y.B.; Zou, D.W.; Wang, J.Y.; Li, J.Y.; Zhou, S.N.; Zhu, Z.Y.; Zhao, X.; Xu, L.P.; Zhang, H.Y. Effects of Chinese Medicine Tong xinluo on Diabetic Nephropathy via Inhibiting TGF- $\beta 1$-Induced Epithelial-to-Mesenchymal Transition. Evid. Based Complement. Altern. Med. 2014, 2014, 123497. [CrossRef]

7. Ma, F.Y.; Gu, C.B.; Li, C.Y.; Luo, M.; Wang, W.; Zu, Y.G.; Li, J.; Fu, Y.J. Microwave-assisted aqueous two-phase extraction of isoflavonoids from Dalbergia odorifera T. Chen leaves. Sep. Purif. Technol. 2013, 115, 136-144. [CrossRef]

8. Sun, S.S.; Zeng, X.; Zhang, D.W.; Guo, S.X. Diverse fungi associated with partial irregular heartwood of Dalbergia odorifera. Sci. Rep. 2015, 5, 8464. [CrossRef]

9. Lee, D.S.; Li, B.; Im, N.K.; Kim, Y.C.; Jeong, G.S. 4,2',5'-Trihydroxy-4'-methoxychalcone from Dalbergia odorifera exhibits anti-inflammatory properties by inducing heme oxygenase-1 in murine macrophages. Int. Immunopharmac. 2013, 16, 114-121. [CrossRef]

10. Lee, D.S.; Kim, K.S.; Ko, W.; Li, B.; Keo, S.; Jeong, G.S.; Oh, H.; Kim, Y.C. The neoflavonoid latifolin isolated from $\mathrm{MeOH}$ extract of Dalbergia odorifera attenuates inflammatory responses by inhibiting NF- $\mathrm{KB}$ activation via Nrf2-mediated heme oxygenase-1 expression. Phytother. Res. 2014, 28, 1216-1223. [CrossRef]

11. Sun, A.H.; Hwang, J.S.; Kang, E.S.; Yoo, T.; Lim, H.H.; Lee, W.J.; Paek, K.S.; Seo, H.G. Ethanol extract of protects skin keratinocytes against ultraviolet $b$-induced photoaging by suppressing production of reactive oxygen species. Biosci. Biotechnol. Biochem. 2015, 79, 760-766.

12. Yu, X.L.; Wang, W.; Yang, M. Antioxidant activities of compounds isolated from Dalbergia odorifera T. Chen and their inhibition effects on the decrease of glutathione level of rat lens induced by UV irradiation. Food Chem. 2007, 104, 715-720. [CrossRef]

13. Zhao, X.B. Studies on the Isolated and Antibacterial Activity of the Flavonoids and Volatile Oil from Dalbergia odorifera T. Chen. Master's Thesis, Tarim University, Tarim, China, June 2012.

14. Yang, Z.H.; Mei, C.; He, X.H.; Sun, X.B. Advance in studies on chemical constitutions, pharmacological mechanism and pharmacokinetic profile of Dalbergia odorifera Lignum. Chin. J. Chin. Mater. Med. 2013, 38, 1679-1683.

15. Liu, R.X.; Wang, Q.; Guo, H.Z.; Li, L.; Bi, K.S.; Guo, D.A. Simultaneous determination of 10 major flavonoids in Dalbergia odorifera by high performance liquid chromatography. J. Pharmaceut. Biomed. 2005, 39, 469-476. [CrossRef] [PubMed]

16. Liu, R.X.; Li, L.; Wang, Q.; Wang, W.; Bi, K.S.; Guo, D.A. Simultaneous determination of nine flavonoids in Dalbergia odorifera by LC. Chromatographia 2005, 61, 409-413. [CrossRef]

17. Li, Y.Y.; Qi, L.K.; Lin, L.; Li, Z.; Kong, J.Y. Simultaneous determination of seven flavonoids in Dalbergiae Odoriferae Lignum by UPLC and principal compounents analysis. Chin. J. Pharm. Anal. 2019, 39, 240-248. 
18. Huang, M.Q.; Zhang, Y.P.; Xu, S.Y.; Xu, W.; Chu, K.D.; Xu, W.; Zhao, H.Y.; Lu, J.J. Identification and quantification of phenolic compounds in Vitex negundo L. var. cannabifolia (Siebold et Zucc.) Hand-Mazz. Using liquid chromatography combined with quadrupole time-of-flight and triple quadrupole mass spectrometers. J. Pharm. Biomed. 2015, 108, 11-20. [CrossRef]

19. Guo, S.; Duan, J.A.; Qian, D.W.; Wang, H.Q.; Tang, Y.P.; Qian, Y.F.; Wu, D.W.; Su, S.L.; Shang, E.X. Hydrophilic interaction ultra-high performance liquid chromatography coupled with triple quadrupole mass spectrometry for determination of nucleotides, nucleosides and nucleobases in Ziziphus plants. J. Chromatogr. A 2013, 1301, 147-155. [CrossRef]

20. Zhao, X.S.; Wei, J.H.; Yang, M.H. Simultaneous analysis of iridoid glycosides and anthraquinones in Morinda officinalis using UPLC-QqQ-MS/MS and UPLC-Q/TOF-MS ${ }^{\mathrm{E}}$. Molecules 2018, 23, 1070. [CrossRef]

21. Zhao, X.S.; Wang, C.H.; Meng, H.; Yu, Z.X.; Yang, M.H.; Wei, J.H. Dalbergia odorifera: A review of its traditional uses, phytochemistry, pharmacology, and quality control. J. Ethnopharmacol. 2020, 248, 112328. [CrossRef]

22. Wang, G.J.; Fu, H.Y.; Ye, W.; Zheng, X.; Xiao, J.C.; Kang, D.; Rao, T.; Shao, Y.H.; Xie, L.; Liang, Y. Comprehensive characterization of the in vitro and in vivo metabolites of ziyuglycoside I in rat microsome, intestinal flora, excretion specimen and fresh tissues based on LC-Q-TOF/MS. J. Pharmaceut. Biomed. 2016, 128, 191-200. [CrossRef]

23. ICH Harmonised Tripartite Guideline. Validation of Analytical procedures: Text and Methodology Q2 (R1). In Proceedings of the International Conference on Harmonization, London, UK, 6 November 2005.

24. Huang, X.; Liu, Y.; Song, F.R.; Liu, Z.Q.; Liu, S.Y. Studies on principal components and antioxidant activity of different Radix astragali samples using high-performance liquid chromatography/electrospray ionization multiple-stage tandem mass spectrometry. Talanta 2009, 78, 1090-1101. [CrossRef] [PubMed]

25. Liu, R.X.; Ye, M.; Guo, H.Z.; Bi, K.S.; Guo, D.A. Liquid chromatography/electrospray ionization mass spectrometry for the characterization of twenty-three flavonoids in the extract of Dalbergia odorifera. Rapid Commun. Mass Spectrom. 2005, 19, 1557-1565. [CrossRef] [PubMed]

26. Zhou, J.L.; Qi, L.W.; Li, P. Herbal medicine analysis by liquid chromatography/time-of-flight mass spectrometry. J. Chromatogr. A 2009, 1216, 7582-7594. [CrossRef] [PubMed]

27. Zhao, C.F.; Liu, Y.Q.; Cong, D.L.; Zhang, H.; Yu, J.J.; Jiang, Y.; Cui, X.Y.; Sun, J.M. Screening and determination for potential $\alpha$-glucosidase inhibitory constituents from Dalbergia odorifera $\mathrm{T}$. Chen using ultrafiltration-LC/ESI-MS ${ }^{\mathrm{n}}$. Biomed. Chromatogr. 2013, 27, 1621-1629. [CrossRef]

28. Chatterjee, A.; Ganguly, D.; Sen, R. New synthesis of 4-phenyl coumarins: Dalbergin and nordalbergin. Tetrahedron 1976, 32, 2407-2408. [CrossRef]

Sample Availability: Samples of the compounds are not available from the authors.

(C) 2020 by the authors. Licensee MDPI, Basel, Switzerland. This article is an open access article distributed under the terms and conditions of the Creative Commons Attribution (CC BY) license (http://creativecommons.org/licenses/by/4.0/). 